\title{
Comparison of single-, double- and triple-combined testing, including Pap test, HPV DNA test and cervicography, as screening methods for the detection of uterine cervical cancer
}

\author{
JU HEE KIM ${ }^{1}$, IN-WOOK KIM ${ }^{1}$, YONG-WAN KIM ${ }^{1}$, DONG CHUN PARK ${ }^{2}$, YONG WOOK KIM $^{2}$, \\ KEUN-HO LEE ${ }^{2}$, TAE-GYU AHN ${ }^{3}$, SEI-JUN HAN ${ }^{3}$ and WOONG SHICK AHN ${ }^{2}$ \\ ${ }^{1}$ Catholic Research Institute of Medical Science, ${ }^{2}$ Department of Obstetrics and Gynecology, Seoul St. Mary's Hospital, \\ College of Medicine, The Catholic University of Korea, Seoul 137-701; ${ }^{3}$ Departments of Obstetrics and Gynecology, \\ Chosun University School of Medicine, Gwangju 501-759, Republic of Korea
}

Received September 26, 2012; Accepted December 3, 2012

DOI: 10.3892/or.2013.2257

\begin{abstract}
Cervical cancer is a serious disease that threatens the health of women worldwide. This study compared the sensitivities and false-positive rates of cervical cytology (Pap smear), human papilloma virus (HPV) DNA test, cervicography, first double-combined testing (cervical cytology and HPV DNA test), second double-combined testing (cervical cytology and cervicography) and triple-combined testing (cervical cytology, HPV DNA test and cervicography). The study included 261 patients screened for uterine cervical cancer. All women simultaneously underwent cervical cytology, HPV DNA test and cervicography for uterine cervical cancer screening and colposcopically directed biopsy for diagnostic evaluation. The triple-combined testing was consistently the most sensitive among the cervical screening tests. The second double-combined testing, with a sensitivity rate of $98.1 \%$ was more sensitive than the first double-combined test $(92.3 \%)$. However, cervical cytology was most specific (93.5\%) and showed the highest positive predictive value (77.8\%). The sensitivity of cervical cytology was markedly improved in combination with HPV DNA test and cervicography. Thus, the triple-combined testing, which improves the high false negativity of cervical cytology, may be an effective tool in uterine cervical cancer screening, pending confirmation of the effectiveness in a mass screening study.
\end{abstract}

\section{Introduction}

Cervical cancer is the third most commonly diagnosed cancer and the fourth leading cause of cancer-related death in females

Correspondence to: Professor Woong Shick Ahn, Department of Obstetrics and Gynecology, Seoul St. Mary's Hospital, The Catholic University of Korea, 505 Banpo-dong, Seocho-ku, Seoul 137-701, Republic of Korea

E-mail: ahnws@catholic.ac.kr

Key words: cervical cancer screening, cervical cytology, HPV DNA test, cervicography worldwide (1). To reduce the rate of death from cervical cancer, the early detection of the disease is crucial. Cervical cytology (the Pap smear) has been accepted as the most reliable and cost-effective method of screening for uterine cervical cancer for the past few decades (2). After being developed by Papanicolaou in 1928, the Pap smear has been the screening method of choice in various countries.

Cervical cytology is considered as to be the most realistic and easy method of screening for uterine cervical cancer. However, cervical cytology has a low level of sensitivity (40-50\%) (3). The diagnostic default of cervical cytology has been associated with failure of many countries to decrease the death rate related to cervical cancer (4).

Early detection is achievable in uterine cervical cancer, which has a relatively long precursor stage, and the examination of the uterine cervix is relatively easy, unlike other organs. It is believed that further knowledge by patients and physicians regarding regular and accurate screening, would significantly decrease the incidence of this cancer. Conservative screening methods and their effectiveness and accuracy have recently been proven (5). Cervicography and the human papillomavirus (HPV) DNA test that have been recently developed have shown clinical significance (6-9). Development of a new method for screening uterine cervical cancer using one or a combination of different screening methods has been recently pursued.

In the present study, we evaluated the clinical significance of three different screening methods (cervical cytology, HPV DNA test and cervicography). After performing cervical cytology, cervicography and/or HPV DNA testing in 261 women, the results obtained from different screening combinations (single, double-combined or triple-combined testing) were compared with the data obtained from colposcopic guided biopsy.

\section{Materials and methods}

Study subjects. The study was performed in 261 women who visited the Department of Obstetrics and Gynecology at the Medical College, Chosun University, Gwangju, during a 
selected 1-year period. Age distribution data are provided in Table I.

Screening methods. Cervical cytology was performed using the two-slide technique with a cytoblush at the endocervix and a spatula in the exocervix $(10,11)$. Readings were carried out at the Department of Anatomy, College of Medicine, Chosun University. HPV DNA test was performed using probe B, which detects HPV intermediate- and high-risk oncogene HPV DNA types 16, 18, 31, 33, 35, 45, 51, 52 and 56, using the Hybrid Capture II system (Digene Diagnostics, San Francisco, CA, USA). After inserting a sterilized speculum into the vagina, a Dacron-tipped applicator was used to smear the cervix, transformation zone and posterior fornix. The smear was collected on a viral Pap medium (Digene Diagnositics) and kept frozen at $-20^{\circ} \mathrm{C}$ until use. Each collected cervix specimen was denatured, and hybridization of the isolated singlestranded DNA was carried out using a ribonucleic acid probe (ViraPap probe B) solution composed of the aforementioned high-risk HPV types. Each mixture composed of RNA/DNA hybrids was placed in a capture test tube coated with the antibody of RNA/DNA hybrids and fixed. The resulting RNA/ DNA hybrids were reacted with an alkaline phosphataseconjugated antibody. The unreacted portion of the conjugated antibody was removed by washing, and the dioxetane-based chemiluminescent, Lumi-Phos 530 (Lumigen, Detroit, MI, USA), was added to facilitate emission of light due to alkaline phosphatase activity. The luminescence was measured using a luminometer and was expressed in relative light units. HPV 11 and HPV 16 DNA (10 pg/ml) were used as positive controls for the low-risk HPV probe and cancer-related high-risk HPV probe, respectively. The result was expressed as the ratio obtained by dividing the relative light units of each specimen by the relative light units of the positive control. When the ratio of the specimen was 1.0 , the result was considered to be HPV DNA-positive and when the ratio was $<1.0$, the result was considered to be negative.

Cervicography was carried out using equipment (TeleCervico) from the National Testing Laboratories (NTL), worldwide. The apparatus was composed of a camera body, ring flash and examination light. After inserting a colposcope, the cervix was smeared with $5 \%$ acetic acid for $15 \mathrm{sec}$, again with $5 \%$ acidic acid for $15-20 \mathrm{sec}$ and for a third time with $5 \%$ acidic acid for $15 \mathrm{sec}$. Images were captured twice by focusing on the cervix and moving the camera back and forth. The reading was carried out by sending the images enlarged by X16 to cervigram evaluators at the NTL and Wisconsin University Medical College. The results were assessed as negative, atypical, positive and technically defective, and were reported using a formatted form including the colposcopy results together with the images (Fig. 1).

Diagnostic methods. The diagnostic methods included performing colposcopy following patient consent and colposcopy guided biopsy in all patients. The aforementioned screening method was combined with cervicography (doublecombined testing). The results of the diagnostic method of colposcopy guided biopsy were compared with the results from the double-combined and triple-combined testing (including cervical cytology and HPV DNA test).
Table I. Age distribution.

\begin{tabular}{lc}
\hline Age (years) & No. of women \\
\hline $20-34$ & 86 \\
$35-54$ & 156 \\
$>55$ & 19 \\
Total & 261 \\
\hline
\end{tabular}

\section{Results}

Histologic results of the cervical cytology. Twenty-eight cases classified as atypical squamous cells of undetermined significance (ASCUS) on cervical cytology were excluded from the statistical analysis due to the ambiguity in classification. On cervical cytology, low grade squamous intraepithelial lesions (HGSILs) were classified as positive for malignancy and negative for normal and benign reactive change. According to the results of the biopsy, cervical intraepithelial neoplasia (CIN) I, II and III, and carcinoma in situ (CIS) cancers were classified as abnormal, and normal, metaplasia and inflammation were placed within the normal limit (WNL). The sensitivity of cervical cytology was $87.5 \%$ and its specificity was $93.5 \%$. Its positive predictive value was $77.8 \%$ and negative predictive value was $96.7 \%$ (Tables II and VI).

Histologic results of the HPV DNA test. HPV DNA test was read according to the presence or absence of response using the Hybrid Capture system. The results of the tissue biopsy were classified as the above-mentioned method. The sensitivity of the HPV DNA test was $72.7 \%$ and its specificity was $91.7 \%$. Its positive predictive value was $70.2 \%$ and its negative predictive value was $92.7 \%$ (Tables II and VI).

Histologic results of the cervicography. Based on cervicography, P0, P1, P2 and P3 were classified as positive, and N1 and $\mathrm{N} 2$ were classified as negative. However, 12 atypical cases were excluded from the analysis. Classsification of the biopsy results was carried out using the above method. The sensitivity of the cervicography was $94.3 \%$ and its specificity was $89.8 \%$. Its positive predictive value was $71.4 \%$ and its negative predictive value was $98.3 \%$ (Tables II and VI).

Histologic results of the double-combined testing (cervical cytology and HPV DNA test). Cases with low-grade squamous intraepithelial lesions (LGSILs), HGSILs or malignancy on cervical cytology, or cases with positive results on the HPV DNA test were considered to be positive. Cases with a normal and benign reactive change on cervical cytology and the cases with negative results on the HPV DNA test were considered to be negative. However, we excluded 23 cases that were ASCUS on cervical cytology and, at the same time, were negative for the HPV DNA test. The classification of the biopsy was carried out using the aforementioned method. The sensitivity of this double-combined testing was $92.3 \%$ and its specificity was $86.6 \%$. Its positive predictive value was $65.8 \%$ and its negative predictive value was $97.6 \%$ (Tables III and VI). 
$\square$ NEGATIVE-Repeat the CERVIGRAM ${ }^{\mathrm{TM}}$ picture and pap smear on a routine basis

1. Components of the transformation zone are visible

2. Components of the transformation zone are not visible

$\square$ ATYPICAL-A CERVIGRAM Repeat the CERVIGRAM picture and pap smear are recommended

in 6 or 12 months

1. Components of the transformation zone are visible

2. Components of the transformation zone are not visible

POSITIVE-Colposcopy is recommended

0. Probable normal variant : appearance Warrant colposcopy to exclude significant disease

1. Compatible with low grade lesion:

2. Compatible with high grade lesion:

3. Compatible with cancer

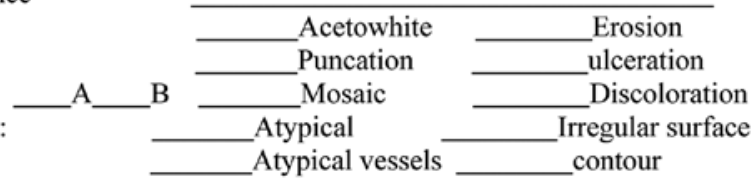

TECHNICALLY DEFECTIVE-See attached notice please retake CERVIGRAM ${ }^{\mathrm{TM}}$ picture

1. View of cervix obscured by : ___ mucus __ blood __ positive of cervix __ other

2. Insufficient acetic acid reaction with reaction is anticipated

3. Other problem: Out of focus Overexposed Underexposed

$\square$ OTHER-(vagina, vulva, fornix, anus-see comments Section)

COMMENTS-

$\square$ CERVGRAM 1M slide(s) is satisfactory for evaluation. The quality could have been improved.

Please note the item(s) marked in the technically defective category above.

Other:

Evaluated By :

Date

Figure 1. Explanation of the categories of the evaluation report and classifications.

Table II. Histologic results of the cervical cytology, HPV DNA test and cervicography.

\begin{tabular}{|c|c|c|c|}
\hline \multirow[b]{2}{*}{ Test } & \multicolumn{2}{|c|}{ Histologic diagnosis } & \multirow[b]{2}{*}{ Total, n (\%) } \\
\hline & Abnormal, n (\%) & WNL, n (\%) & \\
\hline $\begin{array}{l}\text { Cytology }(n=233) \\
\text { [ASCUS }(n=28) \text { was excluded] }\end{array}$ & 48 & 185 & 233 \\
\hline Positive $^{\mathrm{a}}$ & $42(87.5)$ & $12(6.5)$ & $54(30.2)$ \\
\hline Negative $^{\mathrm{d}}$ & $6(12.5)$ & $173(93.5)$ & $179(69.8)$ \\
\hline HPV DNA test $(\mathrm{n}=261)$ & 55 & 206 & 261 \\
\hline Positive $^{c}$ & $40(72.7)$ & $17(8.3)$ & $57(27.9)$ \\
\hline Negative & $15(27.3)$ & $189(91.7)$ & $204(78.1)$ \\
\hline $\begin{array}{l}\text { Cervicography }(\mathrm{n}=249) \\
\text { [Atypical }(\mathrm{n}=12) \text { was excluded] }\end{array}$ & 53 & 196 & 249 \\
\hline Positive $^{d}$ & $50(94.3)$ & $20(10.2)$ & $70(28.1)$ \\
\hline Negative $^{e}$ & $3(5.7)$ & $176(89.8)$ & $179(71.9)$ \\
\hline
\end{tabular}

Histologic results of the double-combined testing (cervical cytology and cervicography). Cases with LGSILs, HGSILs or malignancy on cervical cytology, or cases with positive results on HPV DNA test were considered to be positive. Cases with normal and benign reactive change on cervical cytology and those cases with $\mathrm{N} 1$ and $\mathrm{N} 2$ on cervicography were considered 
Table III. Histologic results of the double-combined testing including cytology and HPV DNA test.

\begin{tabular}{|c|c|c|c|}
\hline \multirow[b]{2}{*}{ Cytology + HPV DNA test } & \multicolumn{2}{|c|}{ Histologic diagnosis } & \multirow[b]{2}{*}{ Total, n $(\%)$} \\
\hline & Abnormal, n (\%) & WNL, n (\%) & \\
\hline Positive $^{\mathrm{a}}$ & $48(92.3)$ & $25 \quad(6.5)$ & $73(30.2)$ \\
\hline Negative $^{b}$ & $4(7.7)$ & $161(93.5)$ & $65(69.8)$ \\
\hline Total & $52(100)$ & $186(100)$ & $238(100)$ \\
\hline
\end{tabular}

aPositive, LGSIL, HGSIL, malignancy; ' Negative, normal, benign reactive change. Abnormal, CIN I, II, III, CIS, cancer; WNL, normal, metaplasia, inflammation. Positive predictive value, $77.8 \%$; negative predictive value, $96.7 \%$. ASCUS ( $\mathrm{n}=28)$ was excluded $(\mathrm{n}=261$ ).

Table IV. Histologic results of the double-combined testing including cytology and cervicography.

\begin{tabular}{|c|c|c|c|}
\hline \multirow[b]{2}{*}{ Cytology + cervicography } & \multicolumn{2}{|c|}{ Histologic diagnosis } & \multirow[b]{2}{*}{ Total, n (\%) } \\
\hline & Abnormal, n (\%) & WNL, n (\%) & \\
\hline Positive $^{\mathrm{a}}$ & $51(98.1)$ & $27(15.3)$ & $73(34.2)$ \\
\hline Negative $^{b}$ & 1 (1.9) & $149(84.7)$ & $150(65.8)$ \\
\hline Total & $52(100)$ & $176(100)$ & $228(100)$ \\
\hline
\end{tabular}

Table V. Histologic results of triple-combined testing including cytology, HPV DNA test, and cervicography.

\begin{tabular}{|c|c|c|c|}
\hline \multirow{2}{*}{ Cytology + HPV DNA test + cervicography } & \multicolumn{2}{|c|}{ Histologic diagnosis } & \multirow[b]{2}{*}{ Total, n (\%) } \\
\hline & Abnormal, n (\%) & WNL, n (\%) & \\
\hline Positive $^{\mathrm{a}}$ & $54(100)$ & $32(17.8)$ & $86(36.8)$ \\
\hline Negative $^{b}$ & $0 \quad(0)$ & $148(82.2)$ & $148(63.2)$ \\
\hline Total & $54(100)$ & $180(100)$ & $234(100)$ \\
\hline
\end{tabular}

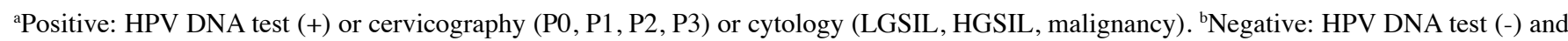
cervicography (N1 or N2) and cytology (normal, benign reactive change). Abnormal, CIN I, II, III, CIS, cancer; WNL, normal, metaplasia, inflammation, infection; Positive predictive value, $62.8 \%$; negative predictive value, $100 \%$. Cases $(\mathrm{n}=22)$ showing an HPV DNA test $(-)$, ASCUS, and N1, N2, A1, A2 and cases $(\mathrm{n}=5)$ showing A1 or A2, and normal or benign reactive change were excluded.

to be negative. However, we excluded 11 cases that were ASCUS on cervical cytology and, at the same time, were A1 or A2 on cervicography. The classification of the biopsy was carried out using the aforementioned method. The sensitivity of this double-combined testing was $98.1 \%$ and its specificity was $84.7 \%$. Its positive predictive value was $65.4 \%$, and its negative predictive value was $99.3 \%$ (Tables IV and VI).

Histologic results of the triple combined testing (cervical cytology, HPV DNA test and cervicography). Cases with LGSILs, HGSILs or malignancy on cervical cytology; positive cases for the HPV DNA test; or P0, P1, P2 and P3 cases on cervicography were considered to be positive. Cases showing normal and benign reactive change on cervical cytology, negative HPV DNA test cases or N1 and N2 cases according to cervicography were considered to be negative. However, we excluded the following cases from analysis: 22 cases that were ASCUS on cervical cytology, were N1, N2, A1 and A2 on cervicography, and showed a negative result on the HPV DNA test; and 5 cases that were A1 and A2 on cervicography, and showed normal or benign reactive changes on cervical cytology. The classification of the biopsy was carried out using the aforementioned method. The sensitivity of this triplecombined testing was $100 \%$ and its specificity was $82.2 \%$. Its 
Table VI. Comparison of cytology alone, HPV DNA test alone, cervicography alone, cytology + HPV DNA test, cytology + cervicography and cytology + HPV DNA test + cervicography.

\begin{tabular}{lcccc}
\hline & $\begin{array}{c}\text { Sensitiviy } \\
(\%)\end{array}$ & $\begin{array}{c}\text { Specificity } \\
(\%)\end{array}$ & $\begin{array}{c}\text { Positive predictive } \\
\text { value }(\%)\end{array}$ & $\begin{array}{c}\text { Negative predictive } \\
\text { value }(\%)\end{array}$ \\
\hline Cytology & 87.5 & 93.5 & 77.8 & 96.7 \\
HPV DNA test & 72.7 & 91.7 & 70.2 & 92.7 \\
Cervicography & 94.3 & 89.8 & 71.4 & 98.3 \\
Cytology + HPV DNA test & 92.3 & 86.6 & 65.8 & 97.6 \\
Cytology + cervicology & 98.1 & 84.7 & 65.4 & 99.3 \\
Cytology + HPV DNA test + cervicology & 100 & 82.2 & 62.8 & 100 \\
\hline
\end{tabular}

positive predictive value was $62.8 \%$ and its negative predictive value was $100 \%$ (Tables V and VI).

Comparison of the results of the single-, double-combined and triple-combined testing. The sensitivity was the highest for the triple-combined testing (100\%) and was the lowest for the HPV DNA test (72.7\%). The specificity was the highest for the cervical cytology at $93.5 \%$ and was the lowest for the triplecombined testing at $82.2 \%$, but no significant difference was evident compared with the other methods. The false-positive rate (1-sensitivity) was the highest for the HPV DNA test at $27.3 \%$ and was the lowest for the cervical cytology (6.5\%). The positive predictive value was the highest for cervical cytology at $77.8 \%$, and was the lowest for the triple-combined testing at $62.8 \%$ (Table VI).

\section{Discussion}

Despite many efforts to reduce the incidence of uterine cervical cancer, approximately 6,000 patients develop cervical cancer each year in Korea, suggesting a problem in the screening system for this disease (12). Furthermore, uterine cervical cancer, through its patterns of pathology, diagnosis and treatment, is indicative of the backwardness of the country in terms of treatment, and still generates tremendous economic and social loss, necessitating measures to deal with this cancer. Despite the fact that cervical cytology, the currently most important screening method for uterine cervical cancer, has contributed significantly to reduce the incidence of cervical cancer for the past several decades (13-15), the high false-positive rate has resulted in social and legal issues in the diagnosis of cervical cancer due to various problems related with the method of collecting specimens, errors and the cervical cytology itself $(16,17)$.

In 1927, Babes observed cervical cells for the purposes of diagnosing uterine cervical cancer. In 1928, Papanicolaou developed a 'new cancer diagnosis' by microscopically observing cancerous cells from vaginal smears. In 1945, the American Cancer Society approved the use of the Pap smear for the diagnosis of uterine cervical cancer. Since then, this method of screening has become a standard at the national level in many countries in North America and Europe, and has contributed significantly to the decreased incidence of cervical cancer by lowering the incidence and mortality of invasive cervical cancer and helping to prevent metastasis of CIN, the precursor stage of cervical cancer, into full-blown uterine cervical cancer through early detection and treatment $(11,18)$.

Currently, cervical cytology has secured its place as one of the most accurate, easy-to-use and cost-effective methods for the early diagnosis and group screening for gynecologic cancers, particularly uterine cervical cancer. However, cervical cancer still develops in individuals who have been screened due to the high false-positive rate of cervical cytology. The high rate of false positivity of cervical cytology results in the misdiagnosis of many uterine cervical cancer patients. Approximately $30 \%$ of invasive cancer cases and $58 \%$ of precursor lesions of cervical cancer are being misdiagnosed based on cervical cytology screening (19).

Many attempts have been made to increase the accuracy of cervical cytology. Of these, the Bethesda System Cytologic Diagnostic Criteria are well-recognized (20). Yet, an increasing number of abnormal smear samples ensues, when compared to previous systems, due to problems related with the classification system. This worries pathologists that more false-positive results may occur along with awareness of increasing HPV infection. This system results in excessive diagnosis and over-treatment due to ambiguity in the concept of ASCUS. Consequently, although the contribution of cervical cytology in reducing the incidence of uterine cervical cancer has been recognized, the need for developing more sensitive screening methods has also been acknowledged.

Based on the latter recognition has come the development of various screening methods including cervicography, HPV DNA test, Papnet, Auto pap, speculoscopy and the use of Polarprobe. These have been proposed as adjunct or substitute screening methods for cervical cytology for the diagnosis of uterine cervical cancer (21). The presently observed $87.5 \%$ sensitivity of cervical cytology was lower compared with those of other screening methods, despite the fact that the screening was carried out only in those patients who visited our hospital, suggesting that not only the method of screening itself using exfoliated cells but, more importantly, the sampling method or reading errors play a major role in decreasing the sensitivity. Additional studies and analyses are also needed, since ASCIS was not included in the present study, which examined only positivity and negativity. In order to improve the high falsepositive rate of cervical cytology, clinical evaluation should be 
carried out in a large and systematic study concerning adjunct screening methods and combined screening methods together with efforts to improve the method of screening and to modify the reporting system.

Currently, the exact mechanism involved in the development of uterine cervical cancer has not been fully elucidated. The widely accepted theory is through sexual contact. HPV is at the center of attention among the infection factors resulting from sexual contact (22). A direct relationship between HPV and the development of uterine cervical cancer has been revealed, and each specific subtype has been identified according to the location and progression of lesions, so that the heterogenicity of the biologic characteristics of HPV has been recognized (23). HPV subtypes 16 and 18 are high-risk viruses associated with the development of uterine cervical cancer, and are detected mainly in HSGILs and invasive cervical cancer. The HPV subtypes 6, 11, 42, 43 and 44 are low-risk viruses and are detected mainly in benign or LGSILs. HPV subtypes $31,33,34,45,51,52$ and 56 are intermediate-risk oncogenic viruses (24). Based on recent active molecular biologic studies, many methods of classifying HPV infection and subtypes have been proposed, but the actual application of these methods is limited in actual clinical settings due to their sensitivity and specificity. The recently developed Hybrid Capture System is a non-amplified method and molecular hybridization method, which uses the chemiluminescent characteristic of DNA from smeared cervical cells, to easily determine the presence of HPV, and to distinguish the identified HPV into high-risk subtypes (HPV 16, 18, 31, 33, 35, 45, 51, 52 and 56) or low-risk subtypes (HPV 6, 11, 42, 43 and 44) quantitatively (25).

Believing that the low sensitivity of cervical cytology could be improved by detecting HPV infection through these different screening methods, we used the HPV DNA test as a screening method for uterine cervical cancer and found that the HPV DNA test alone showed a lower sensitivity compared with other methods of screening $(72.7 \%$, Table VI) but increased the sensitivity of cervical cytology significantly when combined with cervical cytology (92.3\%, Table VI). However, the sensitivity did not reach that of the combined test of cervicography and cervical cytology (98.1\%, Table VI). Thus, this test could be considered as a screening method after reevaluation of this method through group screening. Unlike the existing cervical cytology, the HPV DNA test is a biologic test that could be used not only to determine the current status of a lesion and its surrounding area but also predict the prognosis of the lesion. Attempts have been made in recent years to predict prognosis through the quantification of HPV DNA.

Cervicography was developed in 1981. It enables screening for cervical cancer by capturing images of the cervix coated with 5\% acidic acid using a special camera and allows the evaluation of the enlarged images (26). Its principle and method of approach are similar to those of vaginography; this poses difficulty when being used for screening for a mass, since it requires evaluators to undergo training for an extended period in order to accumulate the needed experience. Other limitations include a lack of objectivity in reading and requirement of expensive equipments. The equipment used for cerivcography can be easily manipulated, is mobile, and requires a reduced period of time to learn since the required handling is easy for mass screening. Moreover, cervicography, which is a morphologic test as is cervical cytology, offers the benefit of showing objective results and allowing follow-up management since it offers a high intraevaluator reproducibility. This method plays a major role in improving the low sensitivity and high false-positive rate of cervical cytology and has been reported to increase sensitivity for diagnosing cervical cancer when combined with cervical cytology (27).

Campion and Reid diagnosed CIN in $68 \%$ of patients using cervical cytology and in $89 \%$ of patients using cervicography (28). Ferris et al diagnosed CIN in $37.5 \%$ of patients using cervical cytology and in $77.8 \%$ using cervicography, and reported that the sensitivity increased to $8.3 \%$ when cervical cytology and cervicography were combined (29). According to one study that examined 12,000 cases, cervicography was the most sensitive screening method for uterine cervical cancer, and the rate of diagnosis was increased when the two screening tests were combined for mass screening of uterine cervical cancer (30). McKinnon et al diagnosed CIN in $92 \%$ and HPV lesions in $82.4 \%$ of patients (31). Costa et al reported $100 \%$ sensitivity when using cervicography alone (30), which was higher than $94.3 \%$ found in the present study. We found a more sensitive result when using cervicography compared with cervical cytology and $98.1 \%$ sensitivity when using both of these tests, indicating that cervicography is very effective for improving the false-positive rate of cervical cytology.

The problem of a high positive rate and low specificity of cervicography has been pointed out, but the specificity has been improved significantly through improvement in the reporting system (32). Coibion et al reported $99.1 \%$ specificity for cervicography (33), and we found $89.8 \%$ in the present study (Table IV). Among single screening methods, cervicography showed the highest sensitivity (94.3\%, Table VI), which was improved significantly when combined with cervical cytology (98.1\%, Table VI). Thus, the combination of the HPV DNA test and cervicography may be very effective for improving the low sensitivity and the high false-positive rate of cervical cytology.

Nonetheless, the possibility of prescribing unnecessary treatment is high since the specificity and positive predictive value are low, despite a high sensitivity. Additionally, a one-time test could generate more testing and increased medical costs due to the advanced equipment used. Unnecessary tests and an increased cost could result in economic and personnel loss; studies are needed to determine the test interval. For example, additional testing should be performed for those cases that show high-grade lesions according to the results of the HPV DNA test, regardless of the results from cervical cytology or cervicography, or for cases showing more than low-grade lesions according to cervical cytology and cervicography in addition to a positive result of the HPV DNA test. These tests should be performed based on the established retest interval for normal cases. The results of the present study cannot be applied directly for uterine cervical cancer screening since the present study was carried out in patients showing a high incidence, thus, further group studies should be carried out using mass screening. Moreover, further problems that remain to be resolved include regional biases, objectivity in reading, accuracy in diagnostic criteria, economic feasibility, excessive treatment due to high sensitivity, and the bothersome nature 
of the tests themselves. In using the triple-combined testing to screen for uterine cervical cancer, these issues should be resolved through more research and adoption of new measures.

\section{References}

1. Jemal A, Bray F, Center MM, Ferlay J, Ward E and Forman D: Global cancer statistics. CA Cancer J Clin 61: 69-90, 2011.

2. Recommendations for cervical screening 1997. Members of the Working Party on Cervical Screening. N Z Med J 111: 94-98, 1998.

3. Miller AB, Nazeer S, Fonn S, et al: Report on consensus conference on cervical cancer screening and management. Int J Cancer 86: 440-447, 2000.

4. Sarian LO, Derchain S, Shabalova I, et al: Optional screening strategies for cervical cancer using standalone tests and their combinations among low- and medium-income populations in Latin America and Eastern Europe. J Med Screen 17: 195-203, 2010.

5. Soler ME and Blumenthal PD: New technologies in cervical cancer precursor detection. Curr Opin Oncol 12: 460-465, 2000.

6. Schiffman M, Herrero R, Hildesheim A, et al: HPV DNA testing in cervical cancer screening: results from women in a high-risk province of Costa Rica. JAMA 283: 87-93, 2000.

7. Wright TC Jr and Schiffman M: Adding a test for human papillomavirus DNA to cervical-cancer screening. N Engl J Med 348: 489-490, 2003.

8. Bukovic D, Fures R, Speranda Z, et al: Cervicography in evaluation of early detection of the cervical lesions. Coll Antropol 26 (Suppl): 143-147, 2002.

9. Franco ES, Hyppolito SB, Franco RG, et al: Digital cervicography criteria: improving sensitivity in uterine cervical cancer diagnosis. Cad Saude Publica 24: 2653-2660, 2008 (In Portuguese).

10. Boon ME, de Graaff Guilloud JC and Rietveld WJ: Analysis of five sampling methods for the preparation of cervical smears. Acta Cytol 33: 843-848, 1989.

11. Koss LG: The Papanicolaou test for cervical cancer detection. A triumph and a tragedy. JAMA 261: 737-743, 1989.

12. Kim YT: Current status of cervical cancer and HPV infection in Korea. J Gynecol Oncol 20: 1-7, 2009.

13. Jin XW, Sikon A and Yen-Lieberman B: Cervical cancer screening: less testing, smarter testing. Cleve Clin J Med 78: 737-747, 2011.

14. Yang KY: Abnormal pap smear and cervical cancer in pregnancy. Clin Obstet Gynecol 55: 838-848, 2012.

15. Sirovich BE and Welch HG: The frequency of Pap smear screening in the United States. J Gen Intern Med 19: 243-250, 2004.

16. DeMay RM: Should we abandon pap smear testing? Am J Clin Pathol 114 (Suppl): S48-S51, 2000.
17. Mount S, Harmon M, Eltabbakh G, Uyar D and Leiman G: False positive diagnosis in conventional and liquid-based cervical specimens. Acta Cytol 48: 363-371, 2004.

18. Richart RM: Screening. The next century. Cancer 76: 1919-1927, 1995.

19. Giles JA, Hudson E, Crow J, Williams D and Walker P: Colposcopic assessment of the accuracy of cervical cytology screening. Br Med J (Clin Res Ed) 296: 1099-1102, 1988.

20. The 1988 Bethesda System for reporting cervical/vaginal cytological diagnoses. National Cancer Institute Workshop. JAMA 262: 931-934, 1989.

21. Sherris J, Wittet S, Kleine A, et al: Evidence-based, alternative cervical cancer screening approaches in low-resource settings. Int Perspect Sex Reprod Health 35: 147-154, 2009.

22. Gavillon N, Vervaet H, Derniaux E, Terrosi P, Graesslin O and Quereux C: How did I contract human papillomavirus (HPV)? Gynecol Obstet Fertil 38: 199-204, 2010 (In French).

23. Stanley M: Pathology and epidemiology of HPV infection in females. Gynecol Oncol 117: S5-S10, 2010.

24. Scheinfeld N and Lehman DS: An evidence-based review of medical and surgical treatments of genital warts. Dermatol Online J 12: 5, 2006.

25. Schiffman MH, Kiviat NB, Burk RD, et al: Accuracy and interlaboratory reliability of human papillomavirus DNA testing by hybrid capture. J Clin Microbiol 33: 545-550, 1995.

26. Wright TC Jr, Denny L, Kuhn L and Goldie S: Use of visual screening methods for cervical cancer screening. Obstet Gynecol Clin North Am 29: 701-734, 2002.

27. Howard M, Sellors JW, Lytwyn A, Roth P and Mahony JB: Combining human papillomavirus testing or cervicography with cytology to detect cervical neoplasia. Arch Pathol Lab Med 128: $1257-1262,2004$.

28. Campion MJ and Reid R: Screening for gynecologic cancer. Obstet Gynecol Clin North Am 17: 695-727, 1990.

29. Ferris DG, Payne P, Frisch LE, Milner FH, diPaola FM and Petry LJ: Cervicography: adjunctive cervical cancer screening by primary care clinicians. J Fam Pract 37: 158-164, 1993.

30. Schiffman MH, Bauer HM, Hoover RN, et al: Epidemiologic evidence showing that human papillomavirus infection causes most cervical intraepithelial neoplasia. J Natl Cancer Inst 85: 958-964, 1993.

31. McKinnon KJ, Ford RM and Hunter JC: Comparison of cytology and cervicography in screening a high risk Australian population for cervical human papillomavirus and cervical intraepithelial neoplasia. Aust N Z J Obstet Gynaecol 33: 176-179, 1993.

32. Hall JB: Can cervicography be improved? Am J Obstet Gynecol 188: 854, 2003.

33. Coibion M, Autier P, Vandam P, et al: Is there a role for cervicography in the detection of premalignant lesions of the cervix uteri? Br J Cancer 70: 125-128, 1994 